In Situ Generation of 3,3,3-Trifluoropropionaldehyde and Its Use for Carbon-Carbon Bond Forming Reactions

Takashi Yamazaki, Rei Kobayashi, Tomoya Kitazume, and Toshio Kubota

\title{
List of Content
}

Experimental details

S2

TABLE 2. Cartesian coordinates of compound $\mathbf{6 a}$

S6 


\section{Experimental section}

General Methods. Unless otherwise noted, materials were obtained from commercial suppliers and were used without further purification. All manipulations involving air-sensitive materials were performed under argon, with such materials being exposed only to thoroughly dried and degassed solvents. Anhydrous ether, THF and $\mathrm{CH}_{2} \mathrm{Cl}_{2}$ were purchased and were used without further purification.

${ }^{1} \mathrm{H}$ NMR (300 MHz) and ${ }^{13} \mathrm{C}$ NMR $(75 \mathrm{MHz})$ spectra were recorded in $\mathrm{CDCl}_{3}$ unless otherwise noted. Chemical shifts were reported in parts per million (ppm) downfield from internal tetramethylsilane $\left(\mathrm{Me}_{4} \mathrm{Si}\right) .{ }^{19} \mathrm{~F}$ NMR spectra $(282 \mathrm{MHz})$ were recorded in $\mathrm{CDCl}_{3}$ unless otherwise noted. Chemical shifts were reported in ppm downfield from internal hexafluorobenzene $\left(\mathrm{C}_{6} \mathrm{~F}_{6}\right)$. Data were tabulated in the following order: multiplicity (s, singlet; $d$, doublet; $t$, triplet; q, quartet; quint, quintet; sex, sextet; sep, septet; m, multiplet; br, broad peak), number of protons, coupling constants (in hertz). Infrared (IR) spectra were obtained as thin films on $\mathrm{NaCl}$ plates, and all spectra were reported in wave numbers $\left(\mathrm{cm}^{-1}\right)$. Column chromatography was conducted with silica gel by using a mixture of hexanes and ethyl acetate $(\mathrm{v} / \mathrm{v})$.

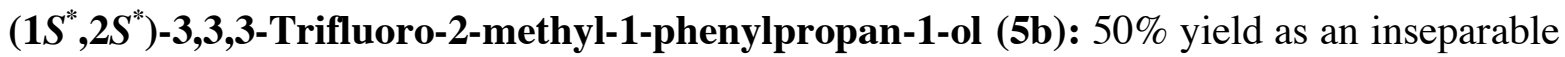
86:14 anti:syn diastereomer mixture. Rf 0.59 (Hex:AcOEt=10:3); ${ }^{13} \mathrm{C}$ NMR $\delta 6.0$ (q, J=2.6 Hz; major), 10.6 (q, $J=3.2 \mathrm{~Hz}$; minor), 44.8 (q, $J=24.3 \mathrm{~Hz}$; minor), 45.2 (q, $J=24.2 \mathrm{~Hz}$; major), 70.5 (q, $J=2.9 \mathrm{~Hz}$; major), 74.0 (q, $J=2.0 \mathrm{~Hz}$; minor), 125.1 (q, $J=3.8 \mathrm{~Hz}$ ), 125.5, 126.8, 127.6 (q, $J=280.0$ Hz; major), 127.6, 128.3, 128.4, 128.5, 128.6, 129.5, 131.6 (q, J=1.4 Hz), 140.8 (minor), 141.4 (major); IR (neat) v 3423, 2996, 2953, 1638, 1600, 1498, 1466, 1371, 1325, 1263, 1172, 1133, 1081, 988, 753, $701 \mathrm{~cm}^{-1}$. Major isomer: ${ }^{1} \mathrm{H}$ NMR $\delta 1.08(3 \mathrm{H}, \mathrm{d}, J=7.1 \mathrm{~Hz}), 2.08(1 \mathrm{H}, \mathrm{bs}), 2.45$ $(1 \mathrm{H}$, dqq, $J=2.5,7.1,9.6 \mathrm{~Hz}), 5.20(1 \mathrm{H}, \mathrm{d}, J=2.2 \mathrm{~Hz}), 7.19-7.63(5 \mathrm{H}, \mathrm{m}) ;{ }^{19} \mathrm{~F}$ NMR $\delta 91.6(\mathrm{~d}$, $J=9.5 \mathrm{~Hz})$. Minor isomer: ${ }^{1} \mathrm{H}$ NMR $\delta 0.85(3 \mathrm{H}, \mathrm{d}, J=7.1 \mathrm{~Hz}), 2.30(1 \mathrm{H}, \mathrm{bs}), 2.62$ (1 H, qquint, $J=7.1,8.5 \mathrm{~Hz}), 4.79(1 \mathrm{H}, \mathrm{d}, J=8.3 \mathrm{~Hz}), 7.19-7.63(5 \mathrm{H}, \mathrm{m}) ;{ }^{19} \mathrm{~F}$ NMR $\delta 93.1$ (d, $\left.J=8.6 \mathrm{~Hz}\right)$.

$\left(3 S^{*}, 4 S^{*}\right)-5,5,5-T r i f l u o r o-4-m e t h y l-1-p h e n y l p e n t-1-y n-3-o l \quad(5 c): \quad 66 \%$ yield as an inseparable 72:28 anti:syn diastereomer mixture. Rf 0.38 (Hex:AcOEt=10:3); IR (neat) v 3422, 2933, 2362, 2344, 2227, 1719, 1599, 1560, 1543, 1508, 1491, 1466, 1444, 1376, 1269, 1173, 1138, 1027, 973, 917, 757, $690 \mathrm{~cm}^{-1}$. Anal. Calcd for $\mathrm{C}_{12} \mathrm{H}_{11} \mathrm{~F}_{3} \mathrm{O}: \mathrm{C}, 63.16 ; \mathrm{H}, 4.86$. Found: C, 62.80; $\mathrm{H}, 5.19$. Major isomer: ${ }^{1} \mathrm{H}$ NMR $\delta 1.38(3 \mathrm{H}, \mathrm{d}, J=7.4 \mathrm{~Hz}), 2.07(1 \mathrm{H}, \mathrm{d}, J=5.8 \mathrm{~Hz}), 2.59(1 \mathrm{H}$, dqq, $J=3.2,7.1,9.6 \mathrm{~Hz}), 4.99(1 \mathrm{H}, \mathrm{dd}, J=3.0,5.8 \mathrm{~Hz}), 7.23-7.62(5 \mathrm{H}, \mathrm{m}) ;{ }^{13} \mathrm{C} \mathrm{NMR} \delta 7.8(\mathrm{q}, J=2.6$ $\mathrm{Hz}), 44.2$ (q, $J=25.1 \mathrm{~Hz}), 61.2$ (q, $J=3.3 \mathrm{~Hz}), 86.0,86.7,126.9$ (q, $J=280.0 \mathrm{~Hz}), 128.2,128.5,128.7$, 131.6; ${ }^{19} \mathrm{~F}$ NMR $\delta 92.0(\mathrm{~d}, J=8.6 \mathrm{~Hz})$. Minor isomer: ${ }^{1} \mathrm{H}$ NMR $\delta 1.38(3 \mathrm{H}, \mathrm{d}, J=7.4 \mathrm{~Hz}), 2.16(1$ $\mathrm{H}, \mathrm{d}, J=5.0 \mathrm{~Hz}), 2.59(1 \mathrm{H}, \mathrm{dqq}, J=3.2,7.1,9.6 \mathrm{~Hz}), 4.89(1 \mathrm{H}, \mathrm{t}, J=5.2 \mathrm{~Hz}), 7.23-7.62(5 \mathrm{H}, \mathrm{m}) ;{ }^{19} \mathrm{~F}$ NMR $\delta 91.9(\mathrm{~d}, J=8.6 \mathrm{~Hz})$.

$\left(3 S^{*}, 4 S^{*}\right)-5,5,5-T r i f l u o r o-4-m e t h y l-1-p h e n y l p e n t-1-o n-3-o l \quad(6 a): 84 \%$ yield as an inseparable 61:39 anti:syn diastereomer mixture. Rf 0.32 (Hex:AcOEt=2:1); IR (neat) v 3509, $3063,2926,2362,2344,1678,1598,1582,1450,1269,1170,934,754,688 \mathrm{~cm}^{-1}$. Anal. Calcd for 
$\mathrm{C}_{12} \mathrm{H}_{13} \mathrm{~F}_{3} \mathrm{O}_{2}: \mathrm{C}, 58.54 ; \mathrm{H}, 5.32$. Found: $\mathrm{C}, 58.90 ; \mathrm{H}$, 5.77. Major isomer: ${ }^{1} \mathrm{H}$ NMR $\delta 1.27(3 \mathrm{H}$, d, $J=7.1 \mathrm{~Hz}), 2.39(1 \mathrm{H}, \mathrm{ddq}, J=4.3,6.9,9.2 \mathrm{~Hz}), 3.17(1 \mathrm{H}, \mathrm{dd}, J=3.4,17.7 \mathrm{~Hz}), 3.26(1 \mathrm{H}, \mathrm{d}, J=3.8$ $\mathrm{Hz}), 3.27(1 \mathrm{H}, \mathrm{dd}, J=8.7,17.7 \mathrm{~Hz}), 4.54(1 \mathrm{H}$, dddd, $J=3.5,4.2,4.2,8.5 \mathrm{~Hz}), 7.47-7.98$ (5 H, m); ${ }^{13} \mathrm{C}$ NMR $\delta 8.3$ (q, $\left.J=2.9 \mathrm{~Hz}\right), 42.6$ (q, $\left.J=1.2 \mathrm{~Hz}\right), 42.8(\mathrm{q}, J=25.0 \mathrm{~Hz}), 65.6$ (q, $\left.J=2.3 \mathrm{~Hz}\right), 127.4$ (q, $J=279.7 \mathrm{~Hz}), 128.0,128.6,133.7,136.3,199.5 ;{ }^{19} \mathrm{~F}$ NMR $\delta 92.3(\mathrm{~d}, J=9.5 \mathrm{~Hz})$. Minor isomer: ${ }^{1} \mathrm{H}$ NMR $\delta 1.25(3 \mathrm{H}, \mathrm{d}, J=7.1 \mathrm{~Hz}), 2.63(1 \mathrm{H}, \mathrm{ddq}, J=4.4,7.1,9.9 \mathrm{~Hz}), 3.14(1 \mathrm{H}, \mathrm{dd}, J=9.6,17.9 \mathrm{~Hz})$, $3.27(1 \mathrm{H}, \mathrm{dd}, J=2.5,17.9 \mathrm{~Hz}), 3.58(1 \mathrm{H}, \mathrm{d}, J=3.6 \mathrm{~Hz}), 4.51-4.59(1 \mathrm{H}, \mathrm{m}), 7.43-7.95(5 \mathrm{H}, \mathrm{m}) ;{ }^{13} \mathrm{C}$ NMR $\delta 8.6(q, J=2.9 \mathrm{~Hz}), 40.6$ (q, $J=1.4 \mathrm{~Hz}), 42.8$ (q, $J=25.0 \mathrm{~Hz}), 65.9$ (q, $J=2.3 \mathrm{~Hz}), 127.2$ (q, $J=282.0 \mathrm{~Hz}$ ), 128.0, 128.6, 133.7, 136.3, 200.1; ${ }^{19} \mathrm{~F}$ NMR $\delta 92.8$ (d, $J=9.5 \mathrm{~Hz}$ ).


inseparable 71:29 anti:syn diastereomer mixture. Rf 0.58 (Hex:AcOEt=2:1); IR (neat) v 3511, 2988, 2360, 2345, 1734, 1653, 1560, 1465, 1374, 1267, 1173, 1143, 1115, 1087, 1022, $991 \mathrm{~cm}^{-1}$. Anal. Calcd for $\mathrm{C}_{9} \mathrm{H}_{13} \mathrm{~F}_{3} \mathrm{O}_{3}: \mathrm{C}, 44.86 ; \mathrm{H}, 6.12$. Found: $\mathrm{C}, 44.68 ; \mathrm{H}, 6.03$. Major isomer: ${ }^{1} \mathrm{H}$ NMR $\delta 1.21(3 \mathrm{H}, \mathrm{d}, J=7.1 \mathrm{~Hz}), 1.29(3 \mathrm{H}, \mathrm{t}, J=7.1 \mathrm{~Hz}), 2.24-2.32(1 \mathrm{H}, \mathrm{m}), 2.50(1 \mathrm{H}, \mathrm{dd}, J=3.6$, $16.8 \mathrm{~Hz}), 2.61(1 \mathrm{H}, \mathrm{dd}, J=9.1,16.8 \mathrm{~Hz}), 2.97(1 \mathrm{H}, \mathrm{d}, J=4.2 \mathrm{~Hz}), 4.19(2 \mathrm{H}, \mathrm{q}, J=7.1 \mathrm{~Hz}), 4.32-4.39$ $(1 \mathrm{H}, \mathrm{m}) ;{ }^{13} \mathrm{C}$ NMR $\delta 7.4$ (q, J=2.6 Hz), 14.0, 39.2, 42.4 (q, $\left.J=25.0 \mathrm{~Hz}\right), 60.8,65.3$ (q, J=2.3 Hz), 127.2 (q, $J=279.5 \mathrm{~Hz}), 171.8 ;{ }^{19} \mathrm{~F}$ NMR $\delta 91.9(\mathrm{~d}, J=9.5 \mathrm{~Hz})$. Minor isomer: ${ }^{1} \mathrm{H}$ NMR $\delta 1.16(3 \mathrm{H}$, t, $J=7.1 \mathrm{~Hz}), 1.29(3 \mathrm{H}, \mathrm{d}, J=7.2 \mathrm{~Hz}), 2.24-2.32$ (1 H, m), $2.51(1 \mathrm{H}, \mathrm{dd}, J=9.4,15.4 \mathrm{~Hz}), 2.54-2.65$ $(1 \mathrm{H}, \mathrm{m}), 3.34(1 \mathrm{H}, \mathrm{d}, J=3.8 \mathrm{~Hz}), 4.20(2 \mathrm{H}, \mathrm{q}, J=7.1 \mathrm{~Hz}), 4.31-4.39(1 \mathrm{H}, \mathrm{m}) ;{ }^{13} \mathrm{C} \mathrm{NMR} \delta 8.2(\mathrm{q}$, $J=3.1 \mathrm{~Hz}), 13.9,39.2,42.8$ (q, $J=24.8 \mathrm{~Hz}), 60.9,65.9$ (q, $J=2.6 \mathrm{~Hz}), 127.0$ (q, $J=279.1 \mathrm{~Hz}), 172.3$; ${ }^{19}$ F NMR $\delta 92.6(\mathrm{~d}, J=9.5 \mathrm{~Hz})$.

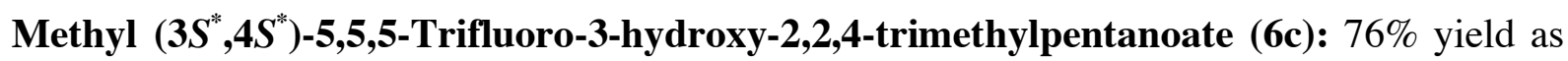
an inseparable 90:10 anti:syn diastereomer mixture. Rf 0.55 (Hex:AcOEt=2:1); IR (neat) v 3511, 2957, 2344, 1719, 1560, 1543, 1474, 1438, 1382, 1333, 1267, 1128, 1032, 990, 946, 917, 856, 803, 772, 694, $648 \mathrm{~cm}^{-1}$. Anal. Calcd for $\mathrm{C}_{9} \mathrm{H}_{15} \mathrm{~F}_{3} \mathrm{O}_{3}: \mathrm{C}, 47.37 ; \mathrm{H}, 6.53$. Found: C, 47.72; H, 6.17. Major isomer: ${ }^{1} \mathrm{H}$ NMR $\delta 1.10(3 \mathrm{H}, \mathrm{d}, J=6.9 \mathrm{~Hz}), 1.21(3 \mathrm{H}, \mathrm{s}), 1.27(3 \mathrm{H}, \mathrm{s}), 2.47-2.30(1 \mathrm{H}, \mathrm{m})$, $2.86(1 \mathrm{H}, \mathrm{d}, J=7.1 \mathrm{~Hz}), 3.73(3 \mathrm{H}, \mathrm{s}), 4.04(1 \mathrm{H}, \mathrm{dd}, J=1.1,7.1 \mathrm{~Hz}) ;{ }^{13} \mathrm{C}$ NMR $\delta 7.4(\mathrm{q}, J=3.0 \mathrm{~Hz})$, 20.9, 23.6, 39.1 (q, $J=24.8 \mathrm{~Hz}$ ), 46.9, 52.4, 72.7 (q, $J=2.9 \mathrm{~Hz}), 127.9$ (q, $J=279.3 \mathrm{~Hz}$ ), 177.2; ${ }^{19} \mathrm{~F}$ NMR $\delta 89.4(\mathrm{~d}, J=9.5 \mathrm{~Hz})$; $\quad$ Minor isomer: ${ }^{1} \mathrm{H}$ NMR $\delta 1.18(3 \mathrm{H}, \mathrm{d}, J=7.1 \mathrm{~Hz}), 1.24(3 \mathrm{H}, \mathrm{s}), 1.30$ $(3 \mathrm{H}, \mathrm{s}), 2.47-2.30(1 \mathrm{H}, \mathrm{m}), 3.24(1 \mathrm{H}, \mathrm{d}, J=8.0 \mathrm{~Hz}), 3.71(3 \mathrm{H}, \mathrm{s}), 4.12(1 \mathrm{H}, \mathrm{d}, J=7.4 \mathrm{~Hz}) ;{ }^{19} \mathrm{~F}$ NMR $\delta 95.0(\mathrm{~d}, J=8.6 \mathrm{~Hz})$.


inseparable 65:35 anti:syn diastereomer mixture. Rf 0.32 (Hex:AcOEt=1:3); IR (neat) v 3448, 2945, 2370, 1794, 1686, 1627, 1277, 1143, 1052, 991, $597 \mathrm{~cm}^{-1}$. Anal. Calcd for $\mathrm{C}_{9} \mathrm{H}_{14} \mathrm{~F}_{3} \mathrm{NO}_{2}: \mathrm{C}$, 45.73; H, 6.62; N, 6.57. Found: C, 45.38; H, 6.79; N, 6.27. Major isomer: ${ }^{1} \mathrm{H}$ NMR $\delta 1.24(3$ H, d, J=7.1 Hz), 2.26-2.39 (1 H, m), $2.52(2 \mathrm{H}, \mathrm{d}, J=5.8 \mathrm{~Hz}), 2.97(3 \mathrm{H}, \mathrm{s}), 3.01(3 \mathrm{H}, \mathrm{s}), 4.24(1 \mathrm{H}$, dq, $J=3.9,5.9 \mathrm{~Hz}), 4.47(1 \mathrm{H}, \mathrm{d}, J=3.6 \mathrm{~Hz}) ;{ }^{13} \mathrm{C}$ NMR $\delta 8.4-8.5(\mathrm{~m}), 36.9$ (q, $\left.J=1.2 \mathrm{~Hz}\right), 35.2,37.0$, 42.6 (q, $J=25.0 \mathrm{~Hz}), 66.0$ (q, $J=2.6 \mathrm{~Hz}), 127.4$ (q, $J=279.5 \mathrm{~Hz}), 171.6 ;{ }^{19} \mathrm{~F}$ NMR $\delta 92.4(\mathrm{~d}, J=9.5$ $\mathrm{Hz})$. Minor isomer: ${ }^{1} \mathrm{H}$ NMR $\delta 1.18(3 \mathrm{H}, \mathrm{d}, J=7.2 \mathrm{~Hz}), 2.41(2 \mathrm{H}, \mathrm{d}, J=4.4 \mathrm{~Hz}), 2.54-2.65(1 \mathrm{H}$, m), $3.01(3 \mathrm{H}, \mathrm{s}), 3.02(3 \mathrm{H}, \mathrm{s}), 4.34(1 \mathrm{H}, \mathrm{ddt}, J=2.7,4.4,9.8 \mathrm{~Hz}), 4.68(1 \mathrm{H}, \mathrm{d}, J=2.8 \mathrm{~Hz}) ;{ }^{13} \mathrm{C}$ 
NMR $\delta 8.3$ (q, $J=2.3 \mathrm{~Hz}), 34.7$ (q, $J=1.4 \mathrm{~Hz}), 35.2,37.0,42.5$ (q, $J=25.0 \mathrm{~Hz}), 66.0$ (q, $J=2.6 \mathrm{~Hz})$, 127.2 (q, $J=279.1 \mathrm{~Hz}), 171.8 ;{ }^{19} \mathrm{~F}$ NMR $\delta 92.7$ (d, $\left.J=9.5 \mathrm{~Hz}\right)$.

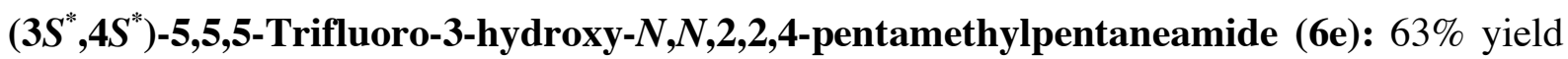
as an inseparable 87:13 anti:syn diastereomer mixture. Rf 0.36 (Hex:AcOEt=1:1); IR (neat) $v$ 3405, 1654, 1604, 1401, 1264, 1171, 1125, $990 \mathrm{~cm}^{-1}$. Anal. Calcd for $\mathrm{C}_{10} \mathrm{H}_{19} \mathrm{~F}_{3} \mathrm{NO}_{2}$ : C, 49.79; $\mathrm{H}$, 7.52; N, 5.81. Found: C, 50.17; H, 7.92; N, 5.64. Major isomer: ${ }^{1} \mathrm{H}$ NMR $\delta 1.19(3 \mathrm{H}, \mathrm{d}, J=7.1$ Hz), $1.32(3 \mathrm{H}, \mathrm{s}), 1.36(3 \mathrm{H}, \mathrm{s}), 2.41(1 \mathrm{H}, \mathrm{dqq}, J=1.8,6.8,10.5 \mathrm{~Hz}), 3.06(6 \mathrm{H}, \mathrm{s}), 3.95(1 \mathrm{H}, \mathrm{dd}$, $J=1.7,6.9 \mathrm{~Hz}$ ), $4.48(1 \mathrm{H}, \mathrm{d}, J=6.9 \mathrm{~Hz}) ;{ }^{13} \mathrm{C}$ NMR $\delta 8.3$ (q, $\left.J=3.1 \mathrm{~Hz}\right), 21.5,23.1,38.4,39.0$ (q, $J=24.4 \mathrm{~Hz}$ ), 46.2, 74.3 (q, $J=2.4 \mathrm{~Hz}), 128.2$ (q, $J=279.4 \mathrm{~Hz}), 177.2 ;{ }^{19} \mathrm{~F}$ NMR $\delta 89.5$ (d, $\left.J=9.5 \mathrm{~Hz}\right)$. Minor isomer: ${ }^{1} \mathrm{H}$ NMR $\delta 1.24(3 \mathrm{H}, \mathrm{d}, J=7.1 \mathrm{~Hz}), 1.36(3 \mathrm{H}, \mathrm{s}), 1.40(3 \mathrm{H}, \mathrm{s}), 2.48-2.61(1 \mathrm{H}, \mathrm{m})$, 3.05 (6 H, s), 3.60 ( $1 \mathrm{H}, \mathrm{dd}, J=4.4,8.5 \mathrm{~Hz}), 4.80(1 \mathrm{H}, \mathrm{d}, J=8.5 \mathrm{~Hz}) ;{ }^{13} \mathrm{C}$ NMR $\delta 13.9$ (q, $\left.J=3.2 \mathrm{~Hz}\right)$, 18.2, 19.2, 23.0, 40.8 (q, $J=24.0 \mathrm{~Hz}), 38.4,80.6(\mathrm{~m}), 127.7$ (q, $J=279.7 \mathrm{~Hz}), 177.5 ;{ }^{19} \mathrm{~F}$ NMR $\delta 94.9$ (d, $J=8.6 \mathrm{~Hz}$ ).

1,1,1-Trifluoro-2-methyl-3-[(trimethylsilyl)oxy]-5-phenylpentane (7): To an ethereal solution $(5 \mathrm{~mL})$ of 2-phenylethyl 2-methyl-3,3,3-trifluoropropionate (246 $\mathrm{mg}, 1.00 \mathrm{mmol})$ was added at $-78{ }^{\circ} \mathrm{C} \mathrm{a} 1 \mathrm{~mol} / \mathrm{L}$ hexane solution of DIBAL-H $(1.05 \mathrm{~mL}, 1.05 \mathrm{mmol})$ and the mixture was stirred for $0.5 \mathrm{~h}$ at that temperature. $0.27 \mathrm{~mL}(1.5 \mathrm{mmol})$ of TMSOTf and $0.24 \mathrm{~mL}(3.0 \mathrm{mmol})$ of pyridine were added successively to this mixture and stirring was continued for $1.5 \mathrm{~h}$ at $0{ }^{\circ} \mathrm{C}$. Quenched the reaction by addition of $0.1 \mathrm{~mL}$ of $\mathrm{H}_{2} \mathrm{O}$ and $\mathrm{MgSO}_{4}$ was added. Filtration and evaporation gave a crude oil containing solid material which was remover by filtration after addition of $n$-hexane. Evaporation of the volatiles furnished 7 in $72 \%$ yield (determined by ${ }^{19} \mathrm{~F}$ NMR) as an inseparable 69:31 diastereomer mixture, which was not subjected for further purification. Rf 0.57 (Hex:AcOEt=10:1); IR (neat) v 3439, 2957, 2872, 1255, 1178, 1140, 1086, $879,841,757 \mathrm{~cm}^{-1}$. Major isomer: ${ }^{1} \mathrm{H}$ NMR $\delta 0.14(9 \mathrm{H}, \mathrm{s}), 1.13(3 \mathrm{H}, \mathrm{d}, J=7.2 \mathrm{~Hz}), 2.50-2.24(1$ H, m), 2.89 (2 H, t, J=7.1 Hz), $3.53(1 \mathrm{H}, \mathrm{dt}, J=7.4,8.9 \mathrm{~Hz}), 3.84(1 \mathrm{H}, \mathrm{dt}, J=7.0,8.8 \mathrm{~Hz}), 4.93(1 \mathrm{H}$, $\mathrm{d}, J=3.6 \mathrm{~Hz}), 7.20-7.35(5 \mathrm{H}, \mathrm{m}) ;{ }^{13} \mathrm{C} \mathrm{NMR} \delta-0.6,0.1,0.2,7.0$ (q, $\left.J=2.9 \mathrm{~Hz}\right), 39.5,44.0$ (q, $J=24.3$ $\mathrm{Hz}), 63.9,95.2$ (q, $J=3.5 \mathrm{~Hz}), 126.8$ (q, $J=280.0 \mathrm{~Hz}), 126.2,128.3,128.6,128.9 ;{ }^{19} \mathrm{~F}$ NMR $\delta 92.3$ (d, $J=8.6 \mathrm{~Hz})$. Minor isomer: ${ }^{1} \mathrm{H}$ NMR $\delta 0.16(9 \mathrm{H}, \mathrm{s}), 1.13(3 \mathrm{H}, \mathrm{d}, J=7.2 \mathrm{~Hz}), 2.50-2.24(1 \mathrm{H}, \mathrm{m})$, $2.89(2 \mathrm{H}, \mathrm{t}, J=7.1 \mathrm{~Hz}), 3.53(1 \mathrm{H}, \mathrm{dt}, J=7.4,9.2 \mathrm{~Hz}), 3.86(1 \mathrm{H}, \mathrm{dt}, J=7.0,9.1 \mathrm{~Hz}), 4.92(1 \mathrm{H}, \mathrm{d}, J=$ $4.1 \mathrm{~Hz}), 7.20-7.35(5 \mathrm{H}, \mathrm{m}) ;{ }^{13} \mathrm{C}$ NMR $\delta-0.6,0.1,0.2,7.1$ (q, $\left.J=2.6 \mathrm{~Hz}\right), 36.2,45.1$ (q, $\left.J=24.4 \mathrm{~Hz}\right)$, 63.3, 95.0 (q, $J=3.4 \mathrm{~Hz}), 126.7$ (q, $J=279.9 \mathrm{~Hz}), 126.2,128.2,128.6,128.9 ;{ }^{19} \mathrm{~F}$ NMR $\delta 92.4(\mathrm{~d}$, $J=9.5 \mathrm{~Hz})$. 
TABLE 2. Cartesian coordinates of compound 6a

$\begin{array}{llll}\text { atom } & \mathrm{x} & \mathrm{y} & \mathrm{z} \\ \mathrm{F}(1) & 0.3169(1) & -0.18864(4) & 0.0426(2) \\ \mathrm{F}(2) & 0.3312(1) & -0.17138(4) & 0.3062(1) \\ \mathrm{F}(3) & 0.46443(9) & -0.15661(4) & 0.1413(2) \\ \mathrm{O}(1) & 0.5237(1) & 0.03004(5) & 0.3111(2) \\ \mathrm{O}(2) & 0.3494(1) & -0.05513(5) & 0.3953(1) \\ \mathrm{C}(1) & 0.4440(1) & 0.04823(5) & 0.2391(2) \\ \mathrm{C}(2) & 0.3648(1) & 0.00803(6) & 0.1586(2) \\ \mathrm{C}(3) & 0.3820(1) & -0.05093(5) & 0.2218(2) \\ \mathrm{C}(4) & 0.3175(1) & -0.09380(6) & 0.1193(2) \\ \mathrm{C}(5) & 0.3574(1) & -0.15198(6) & 0.1534(2) \\ \mathrm{C}(6) & 0.4241(1) & 0.10905(5) & 0.2314(2) \\ \mathrm{C}(7) & 0.4974(1) & 0.14443(6) & 0.3111(2) \\ \mathrm{C}(8) & 0.4802(2) & 0.20109(7) & 0.3108(3) \\ \mathrm{C}(9) & 0.3914(2) & 0.22299(7) & 0.2305(3) \\ \mathrm{C}(10) & 0.3190(2) & 0.18873(7) & 0.1504(3) \\ \mathrm{C}(11) & 0.3346(1) & 0.13166(6) & 0.1508(2) \\ \mathrm{C}(12) & 0.1964(1) & -0.09043(9) & 0.1479(3) \\ \mathrm{H}(1) & 0.296(1) & 0.0200(7) & 0.184(2) \\ \mathrm{H}(2) & 0.374(1) & 0.0097(7) & 0.038(2) \\ \mathrm{H}(3) & 0.457(1) & -0.0594(5) & 0.210(2) \\ \mathrm{H}(4) & 0.333(1) & -0.0895(6) & 0.001(2) \\ \mathrm{H}(5) & 0.558(1) & 0.1292(7) & 0.368(2) \\ \mathrm{H}(6) & 0.528(1) & 0.2222(8) & 0.366(2) \\ \mathrm{H}(7) & 0.379(1) & 0.2616(9) & 0.228(2) \\ \mathrm{H}(8) & 0.259(2) & 0.2032(8) & 0.096(3) \\ \mathrm{H}(9) & 0.286(1) & 0.1090(7) & 0.093(2) \\ \mathrm{H}(10) & 0.172(1) & -0.0550(8) & 0.103(2) \\ \mathrm{H}(11) & 0.178(2) & -0.0959(9) & 0.266(3) \\ & 0.160(1) & -0.1196(8) & 0.086(2) \\ \mathrm{H}) & -0.0467(8) & 0.454(3)\end{array}$

